\title{
Coordinated RhoA signaling at the leading edge and uropod is required for T cell transendothelial migration
}

\author{
Sarah J. Heasman, 'Leo M. Carlin, ${ }^{1,2}$ Susan Cox, ${ }^{1}$ Tony $\mathrm{Ng}^{1,2}$ and Anne J. Ridley ${ }^{1}$ \\ 'Randall Division of Cell and Molecular Biophysics and 2Richard Dimbleby Department of Cancer Research, King's College London, London SE 1 1UL, England, UK
}

$\mathrm{T}$ ransendothelial migration (TEM) is a tightly regulated process whereby leukocytes migrate from the vasculature into tissues. Rho guanosine triphosphatases (GTPases) are implicated in TEM, but the contributions of individual Rho family members are not known. In this study, we use an RNA interference screen to identify which Rho GTPases affect T cell TEM and demonstrate that RhoA is critical for this process. RhoA depletion leads to loss of migratory polarity; cells lack both leading edge and uropod structures and, instead, have stable narrow protrusions with delocalized protrusions and contractions.
By imaging a RhoA activity biosensor in transmigrating T cells, we find that RhoA is locally and dynamically activated at the leading edge, where its activation precedes both extension and retraction events, and in the uropod, where it is associated with ROCK-mediated contraction. The Rho guanine nucleotide exchange factor (GEF) GEF-H1 contributes to uropod contraction but does not affect the leading edge. Our data indicate that RhoA activity is dynamically regulated at the front and back of $T$ cells to coordinate TEM.

\section{Introduction}

After the capture of leukocytes on blood vessel walls, they polarize and migrate on the endothelial cells (ECs) lining the vessels, which is thought to enable them to find optimal sites for transendothelial migration (TEM; Schenkel et al., 2004; Phillipson et al., 2006). Leukocytes transmigrate either at EC junctions (paracellular TEM) or through individual EC (transcellular TEM; Carman and Springer, 2004; Millán et al., 2006; Vestweber, 2007). These different steps of TEM require the coordinated control of adhesion and the cytoskeleton.

Rho GTPases regulate cytoskeletal dynamics and migration in many diverse cell types (Jaffe and Hall, 2005; Heasman and Ridley, 2008). In T cells and other leukocytes, expression of dominant-negative Rho GTPases or treatment with chemical inhibitors or bacterial toxins has implicated several Rho GTPases in leukocyte migration (Tybulewicz and Henderson, 2009). For example, inhibitors of the closely related Rho isoforms RhoA,

Correspondence to Anne J. Ridley: anne.ridley@kcl.ac.uk

Abbreviations used in this paper: EC, endothelial cell; ERM, ezrin-radixinmoesin; FLIM, fluorescence lifetime imaging microscopy; FRET, fluorescence resonance energy transfer; GEF, guanine nucleotide exchange factor; HUVEC, human umbilical vein EC; MTOC, microtubule-organizing center; $\mathrm{p}-\mathrm{ERM}$, phosphorylated ERM; $\mathrm{p}-\mathrm{MLC}$, phosphorylated myosin light chain; STED, stimulated emission depletion; TEM, transendothelial migration.
RhoB, and RhoC and/or their downstream targets, ROCKs, have been reported to reduce leukocyte adhesion by inhibiting activation of the integrin LFA-1 (Giagulli et al., 2004) and to reduce migration and chemotaxis by inhibiting contraction of the uropod at the rear (Alblas et al., 2001; Vicente-Manzanares et al., 2002; Smith et al., 2003; Worthylake and Burridge, 2003). In knockout mice, Cdc42, Rac1, and Rac2 contribute to the recruitment of leukocytes to inflamed sites (Roberts et al., 1999; Yamauchi et al., 2004; Szczur et al., 2006; Filippi et al., 2007), although their exact role in the individual steps of TEM is not yet defined. Studies using leukocytes from knockout mice have identified roles for Cdc42 and the Rac guanine nucleotide exchange factor (GEF) Tiam1 (Szczur et al., 2006; Gérard et al., 2009) in TEM. However, to date, the roles of the 20 different Rho GTPases in leukocyte TEM have not been systematically addressed. In this study, we use an siRNA screen to identify which Rho GTPases affect $\mathrm{T}$ cell TEM and describe a key role for RhoA in this process.

(c) 2010 Heasman et al. This article is distributed under the terms of an AttributionNoncommercial-Share Alike-No Mirror Sites license for the first six months after the publication date (see http://www.rupress.org/terms). After six months it is available under a Creative Commons License (Attribution-Noncommercial-Share Alike 3.0 Unported license, as described at http://creativecommons.org/licenses/by-nc-sa/3.0/). 


\section{Results and discussion}

RhoA is required for $T$ cell TEM

We initially determined which of the 20 Rho GTPase family members were expressed in primary $\mathrm{T}$ lymphoblasts and the T cell line, CCRF-CEM (CEM). We detected expression of 14 Rho GTPases but not RhoC, RhoV, RhoJ, RhoD, or RhoE/Rnd3. Rnd2 was only detected in CEM cells and not T lymphoblasts (Fig. S1 b). Using a siRNA screen, we examined which of these Rho GTPases affected TEM (Fig. S1, a and c). RhoA depletion had the strongest effect on TEM. The extent of TEM inhibition correlated with the level of RhoA knockdown, as observed with two siRNAs that knocked down RhoA with different efficiencies or with lentivirally delivered shRNA (Fig. 1, a and b; and Fig. S1, c and d). Those cells that did complete TEM did so later than control cells (Fig. $1 \mathrm{c}$ ).

RhoA is highly homologous to RhoB and RhoC (Boureux et al., 2007), but RhoC was not detected in T cells (Fig. S1 b). RhoB knockdown was less efficient, and thus, we cannot rule out a contribution to TEM (Fig. S1 c). The screen also indicated an important role for Rac2, whereas Rac3 knockdown increased TEM (Fig. S1 a). Rac3 has not previously been studied in hemopoietic cells, largely because its expression is highest in neurons (Bolis et al., 2003). However, it has previously been shown to have an opposing effect to Rac1 in neuronal cell adhesion and differentiation (Hajdo-Milasinović et al., 2007). Knockdown of RhoGDI1 was recently reported to lead to reduced levels of RhoA, Rac1, and Cdc42 (Boulter et al., 2010). RhoA depletion did not alter Rac1 or Cdc42 levels significantly in CEM cells, although Rac1 activity was reduced (Fig. S1 e). It is unlikely that this contributes to the TEM inhibition after RhoA knockdown, as Rac1 knockdown only marginally reduced TEM (Fig. S1 a).

\section{Depletion of RhoA prevents $\mathbf{T}$ cell crawling on endothelium}

To determine at which stage RhoA affected TEM, we observed $\mathrm{T}$ cell interactions with EC by time-lapse microscopy. Control siRNA-treated cells rapidly polarized and migrated, with a lamellipodium at the front and uropod at the rear (the uropod is a domain behind the nucleus containing the microtubule-organizing center [MTOC] and signaling and adhesion molecules such as ICAM-3; Sánchez-Madrid and Serrador, 2009). Most transmigrating cells used a paracellular pathway $(75 \pm 4 \%$ SEM of transmigrating cells; $18 \pm 3 \%$ SEM of total cells adhered to EC; Fig. 1 d). RhoA knockdown cells did not polarize or crawl efficiently on EC, and their migratory velocity was much lower than control cells (Fig. 1, d-f; and Video 1). Instead of having a single lamellipodium and uropod, RhoA-depleted cells had narrow protrusions extending from a rounded cell body. Most RhoA-depleted cells had two protrusions, generally at opposite sides of the cell body, but some cells had three or more protrusions (Fig. $1 \mathrm{f}$ and Video 1). These protrusions extended and retracted at the tips but did not move the cell body significantly.

Endothelial ICAM-1 clusters around adherent leukocytes forming transmigratory cups (Barreiro et al., 2002; Carman et al., 2003; Carman and Springer, 2004; van Buul et al., 2007) that are thought to be involved in transmigration (Wittchen, 2009).
RhoA-depleted cells were not deficient in ICAM-1 clustering; indeed, there was an increase in cells associated with ICAM-1 clusters (Fig. $1 \mathrm{~d}$ ), implying that cups might preferentially form around $\mathrm{T}$ cells that are unable to transmigrate. It has been reported that Tiam1-null $\mathrm{T}$ cells have reduced paracellular TEM but are able to switch to transcellular TEM (Gérard et al., 2009). In contrast, RhoA-depleted cells did not switch to transcellular TEM (Fig. $1 \mathrm{~d}$ ), suggesting that RhoA is required for both routes.

\section{RhoA is required for $T$ cell polarization on ECs}

We investigated how RhoA knockdown affected T cell migratory polarity on EC. Polarized cells were identified by their elongated shape and polarized localization of F-actin at the leading edge and the MTOC in the uropod (Fig. 2 a). By 30 min after addition to $\mathrm{EC}$, most control siRNA-transfected $\mathrm{T}$ cells were polarized (Fig. 2 b). RhoA depletion increased the number of unpolarized cells with two or more narrow protrusions and lacking a broad lamellipodium. The MTOC localized in the center of the rounded cell body, and the narrow protrusions contained both F-actin and microtubules (Fig. 2 a). These protrusions moved very slowly in comparison with lamellipodia in control cells (Fig. $2 \mathrm{c}$ and Videos 2 and 4). Some RhoA-depleted cells retained a broad lamellipodium but had an elongated tail and no clear uropod. These cells failed to detach the rear from EC (Fig. $2 \mathrm{c}$ and Video 3). The fraction of rounded cells with multiple protrusions increased with efficiency of RhoA knockdown (Fig. 2 b, RhoA siRNA 1 and 2), suggesting that intermediate RhoA levels are sufficient for cells to extend a lamellipodium but not to maintain the level of actomyosin contractility required for uropod formation, whereas lamellipodia cannot form at lower RhoA levels.

RhoA depletion also affected the localization of other T cell migratory polarity markers. Polarized control cells accumulated the integrin LFA-1 (CD11a/ $\beta 2)$ in the lamellal region and ICAM-3 and phosphorylated ezrin-radixin-moesin proteins (ERM [p-ERM]) in the uropod (Fig. 2 a; Sánchez-Madrid and Serrador, 2009). In RhoA-depleted cells with multiple protrusions, ICAM-3 and LFA-1 were no longer polarized, and p-ERM was not detectable. In RhoA-depleted cells with elongated tails, ICAM-3 was more diffusely localized on the plasma membrane toward the cell rear than in control cells, and LFA-1 localized in the tails as well as lamellae. p-ERM detected by immunofluorescence appeared much reduced, which is consistent with a role for RhoA in stimulating ERM phosphorylation (Matsui et al., 1999).

RhoA is localized to and active at the

leading edge and uropod of crawling and

transmigrating $T$ cells

To understand how RhoA affects migratory polarity and TEM, we examined RhoA localization. RhoA was enriched both at the leading edge and uropod of T cells during crawling and TEM (Fig. 3 a). To image RhoA activity, a RhoA Raichu probe (Yoshizaki et al., 2003) modified to express GFP/RFP (Makrogianneli et al., 2009) was expressed in CEM cells. The fluorescence resonance energy transfer (FRET) efficiency of the probe is determined by 

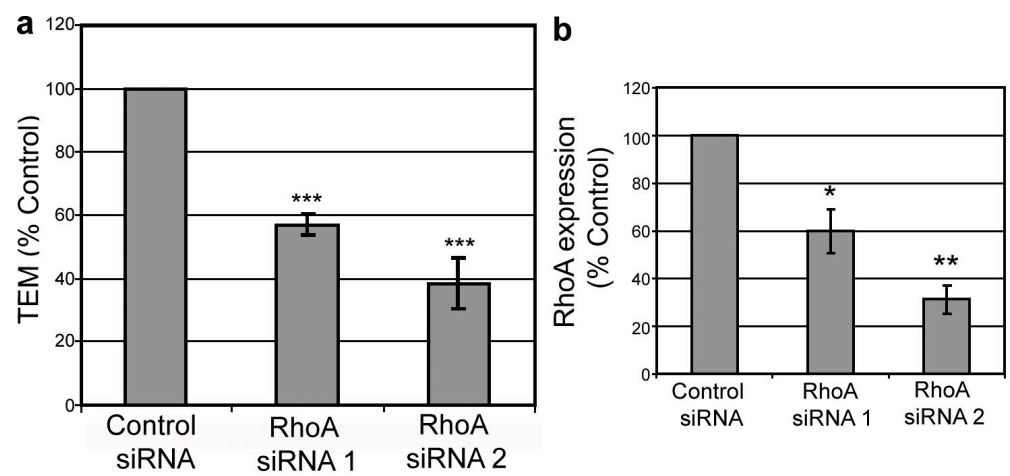

C
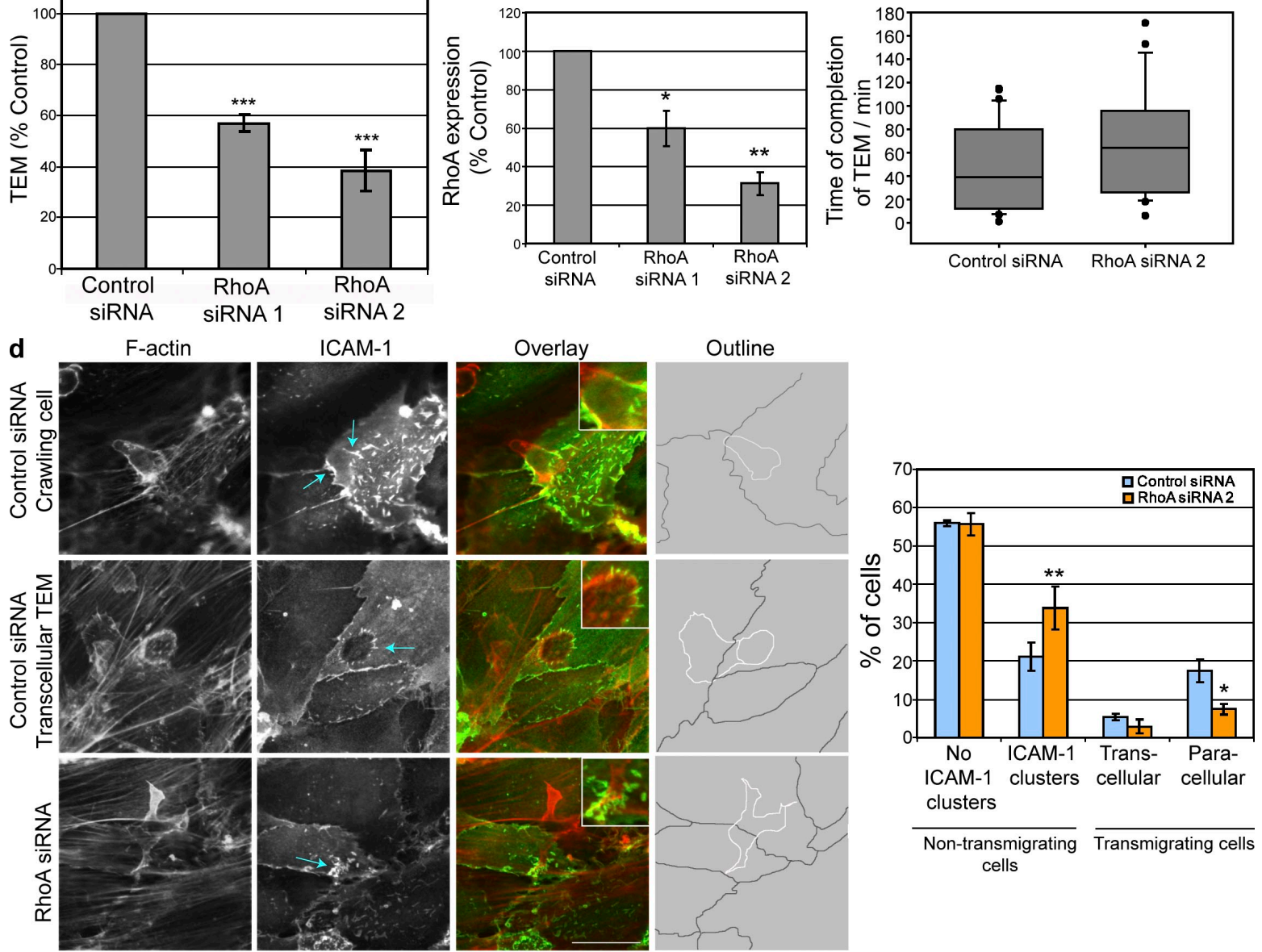

e
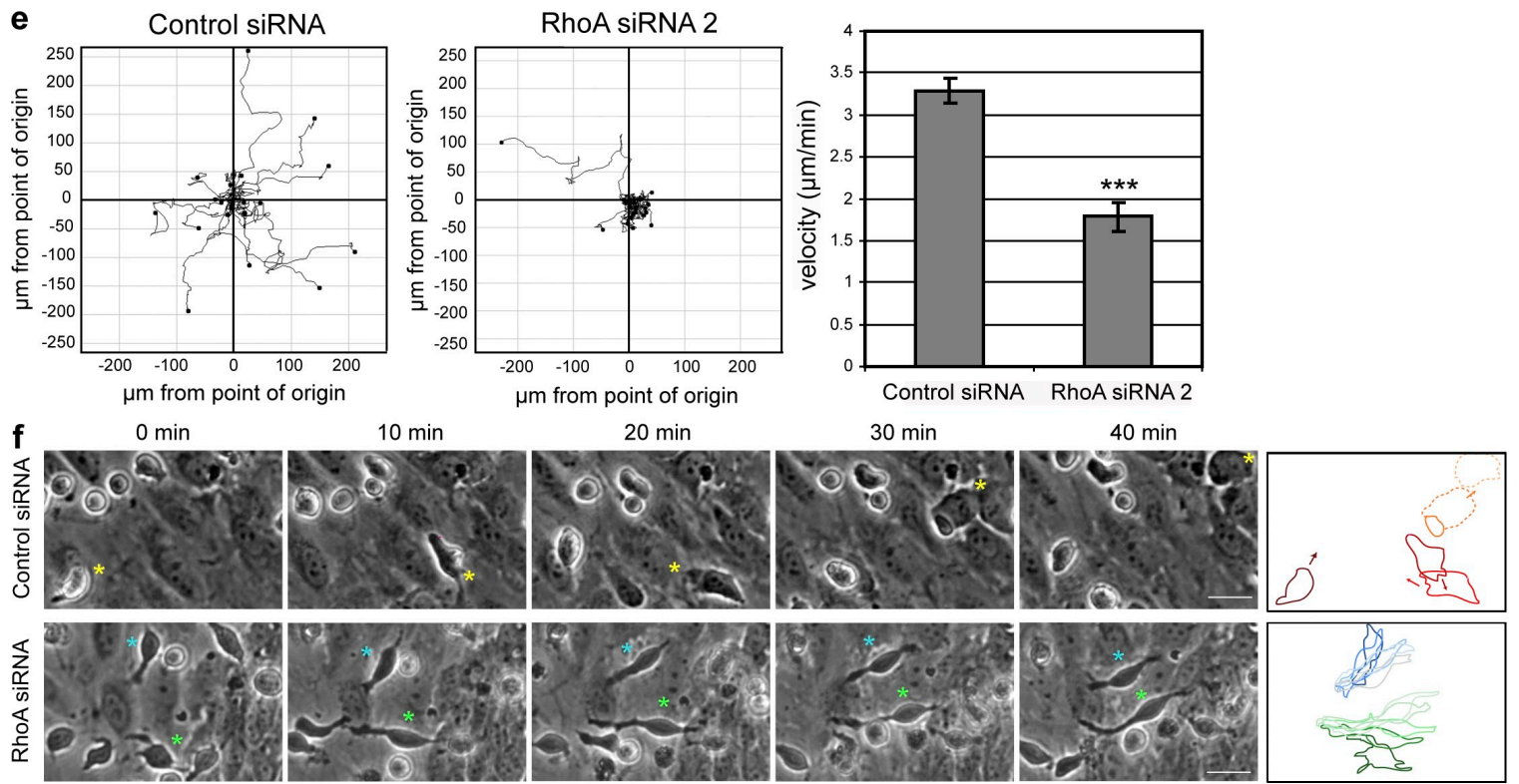

Figure 1. RhoA is required for T cell crawling and TEM. (a) CEM T cell TEM on Transwells ( $n \geq 5$ ). (b) RhoA expression in CEM cells relative to GAPDH $(n=3)$. (c) Time of TEM completion determined by time-lapse microscopy (control siRNA, $n=32$ cells; RhoA siRNA, $n=21$ cells; four experiments). Boxes, median, 25th, and 75th percentiles; whiskers, 5th and 95th percentiles; dots, outliers. (d, left) Endothelial ICAM-1 localization around CEM cells on EC. Arrows, ICAM-1 clusters. ICAM-1 localizes around transmigratory channel (middle) or clusters around T cell processes of RhoA-depleted cells (bottom). (right) Quantification of ICAM-1 clustering around nontransmigrating cells and transmigratory path $(n=4 ; \geq 100$ cells/experiment). Insets: magnification of regions of ICAM-1 clustering indicated by blue arrows. (e, left) Tracks of 20 representative cells on EC from time-lapse videos. (right) Mean velocity of tracked cells $(n=4 ; \geq 38$ cells/experiment). ( $f$ ) Images from time-lapse videos of CEM cells crawling on and transmigrating across EC. (right) Outlines of asterisk-marked control (yellow) and RhoA siRNA (blue and green) cells. Arrows, migration direction of control cells; dashed lines, transmigrated regions. ${ }^{*}, \mathrm{P}<0.05 ;{ }^{*}, \mathrm{P}<0.01 ;{ }^{* *}, \mathrm{P}<0.001$. Error bars indicate mean \pm SEM. Bars, $20 \mu \mathrm{m}$. 


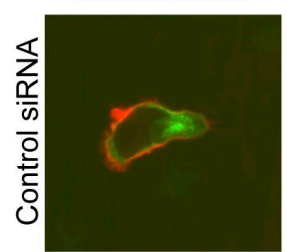

F-actin

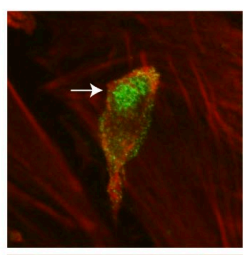

F-actin

ICAM3

\section{Tracker Dye}

p-ERM
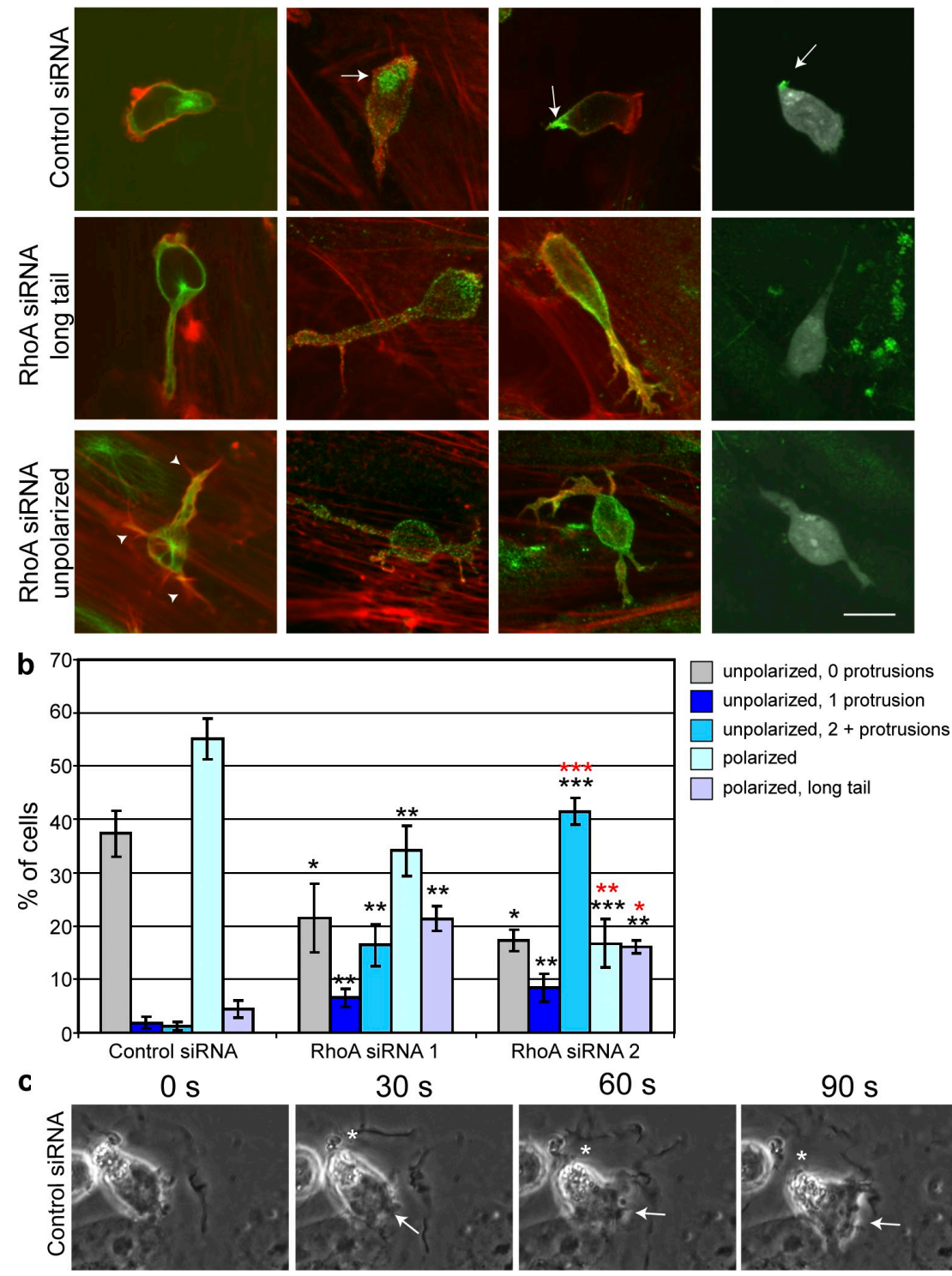

$\square$ polarized

polarized, long tail
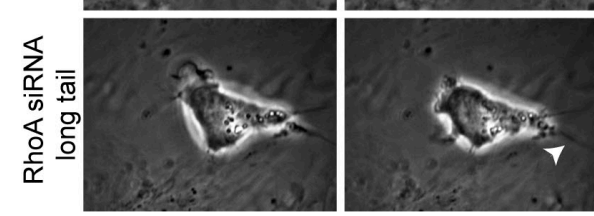

RhoA siRNA 1

RhoA siRNA 2
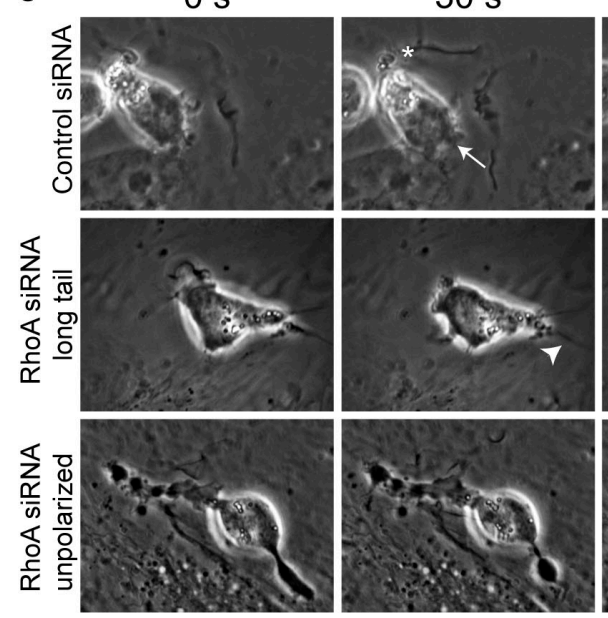

$60 \mathrm{~s}$

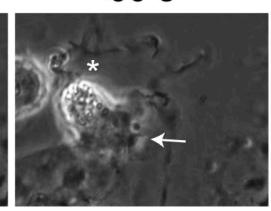

$90 \mathrm{~s}$
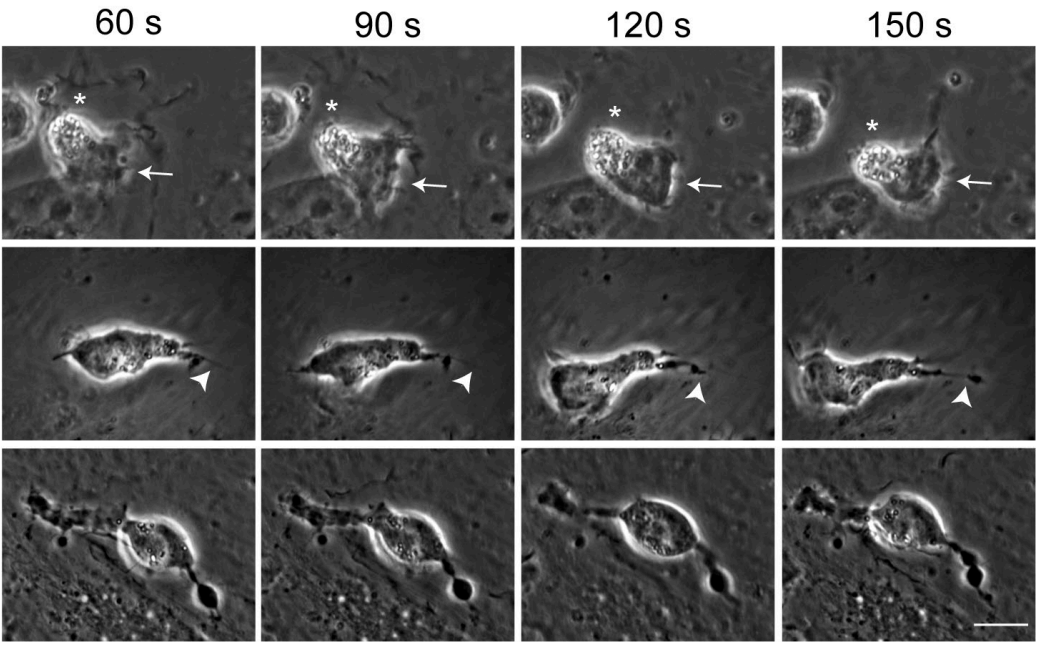

Figure 2. RhoA is required for T cell polarity on ECs. (a) CEM cells on EC were stained for the indicated proteins. Representative RhoA knockdown cells with an elongated tail (middle) or two or more protrusions (bottom). Arrows, polarized protein localization in control cells; arrowheads, F-actin-rich protrusions in RhoA knockdown. (b) CEM cells on EC were scored for the indicated polarity categories $\left(n=3 ; \geq 100\right.$ cells/experiment). ${ }^{*}, P<0.05 ;{ }^{* *}, \mathrm{P}<0.01$; $* * *, P<0.001$; compared with siControl (black asterisks) or siRhoA-1 (red asterisks) determined by Student-Newman-Keuls repeated measure analysis of variance. (c) Images from time-lapse videos. (top) Polarized control cell migrating on EC. Arrows, lamellipodium; * , uropods. (middle) Polarized RhoA knockdown cell. Arrowheads, elongated tails. (bottom) Rounded RhoA knockdown cell with two protrusions. Error bars indicate mean \pm SEM. Bars, $10 \mu m$.

the GTP/GDP loading and thus reports RhoA activity. Localization of the probe was similar to endogenous RhoA (Fig. S2 d). Live cell multiphoton fluorescence lifetime imaging microscopy
(FLIM) was performed to examine the spatiotemporal regulation of RhoA activity during $\mathrm{T}$ cell crawling and TEM. RhoA activity was observed in three regions: at the leading edge at 
a

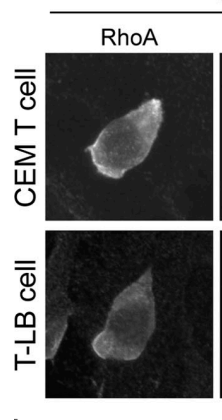

Crawling cells
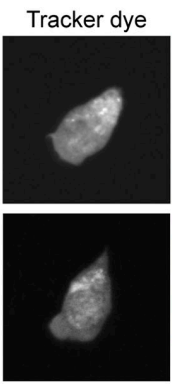
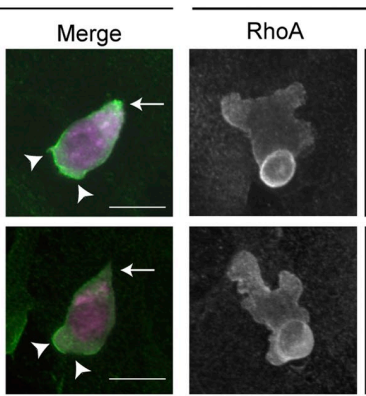

Transmigrating cells
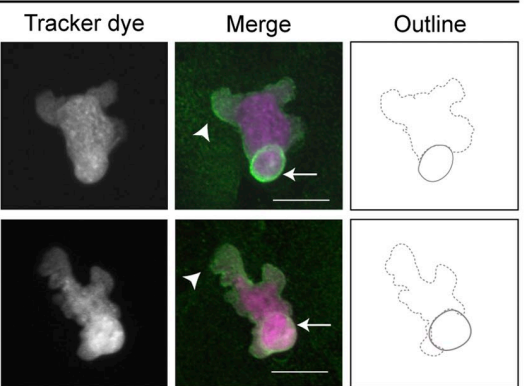

Wild type RhoA probe Lifetime
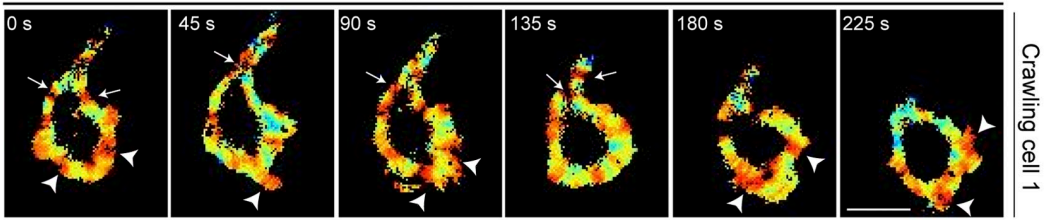

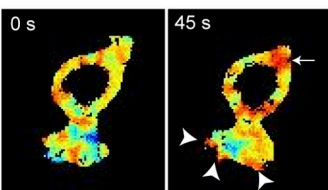

c

Transmigrating cell 1
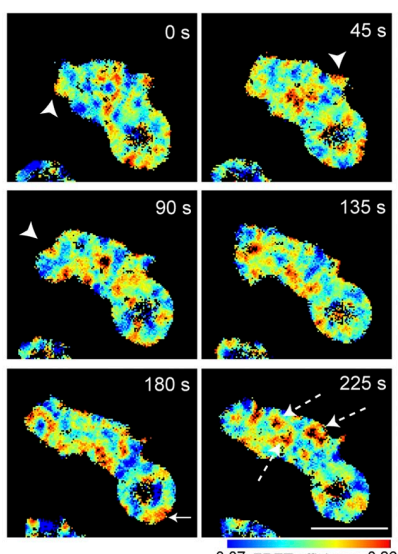

0.07 FRET efficiency 0.2

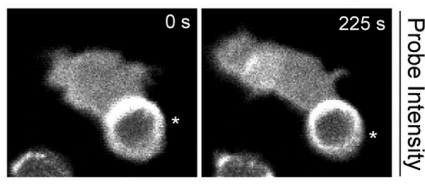

e

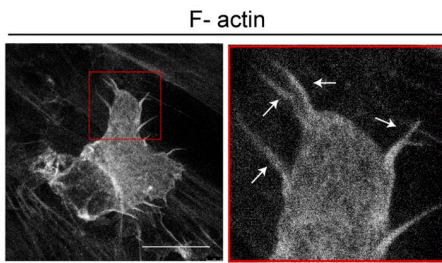

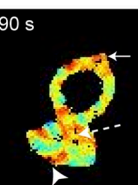

$135 \mathrm{~s}$
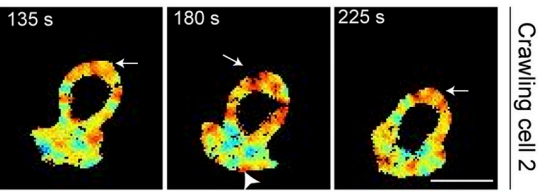

0.07 FRET efficiency 0.26

d Comparison of FRET efficiency values between regions of cell (\% of total number of frames analyzed per cell where RhoA activity was higher at front $v$ side and back $v$ side)

\begin{tabular}{|c|c|c|c|}
\hline & No of frames & & \\
\hline Cell & analyzed & Front $>$ Side & Back>side \\
\hline A & 25 & 80.0 & 52.0 \\
\hline B & 8 & 62.5 & 75.0 \\
\hline C & 12 & 66.7 & 75.0 \\
\hline D & 12 & 66.7 & 75.0 \\
\hline$E$ & 8 & 75.0 & 25.0 \\
\hline $\mathrm{F}$ & 18 & 11.1 & 66.7 \\
\hline
\end{tabular}
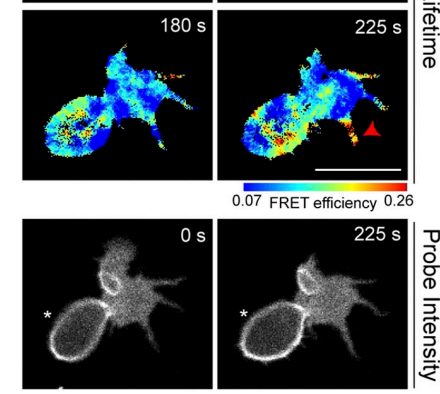

\begin{abstract}
T cell outline
\end{abstract}
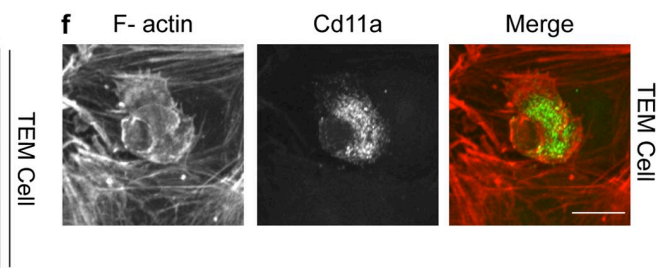

Figure 3. RhoA localizes to and is active at the leading edge and uropod during crawling and TEM. (a) RhoA (green) localization in CEM T cells or T lymphoblasts (T-LB) on EC. Pink, cell tracker dye. Maximum projections of 11-31 confocal z-stack images at 0.5- $\mathrm{mm}$ intervals. RhoA is enriched at the uropod (arrows) and leading edge (arrowheads). (right) Circles, uropods; dashed lines, lamellipodium under EC. (b and c) FLIM images of RhoA Raichu probe (wild type) were acquired every $45 \mathrm{~s}$ as cells migrated on (b) or transmigrated across (c) EC. RhoA activity localizes to the leading edge (white arrowheads), including filopodia during TEM (red arrowheads), the uropod during rear contraction (solid arrows), and in dynamic puncta in the lamellal region (dashed arrows). Probe intensity images identify uropod (asterisks) above and transmigrated lamella below EC. (d) The plasma membrane region of FLIM images from six videos was analyzed to quantify RhoA activity levels (mean FRET efficiency value) in each of four $90^{\circ}$ segments (see Materials and methods). The center of the leading edge is center of the front segment. The sum of values in the front or back segment was compared with the two side segments. (e) F-actin localization in transmigrating CEM cell imaged by STED microscopy. (middle) Magnified image of boxed area shows filopodia extending from the leading edge (arrows). (right) Circle, uropod; dashed line, leading edge under EC. (f) Localization of $\mathrm{Cd} 1 \mathrm{la} / \alpha \mathrm{L}$ integrin (green) and F-actin (red) during TEM 30 min after addition of CEM cells to EC. Bars, $10 \mu \mathrm{m}$. 
regions of protrusion, at the uropod, and, in some cells where we imaged the basal plasma membrane, in dynamic puncta in the lamella behind the leading edge (Fig. 3, b and c; and Videos 5-8). Peaks of RhoA activity on the plasma membrane were higher at the leading edge compared with sides $(\mathrm{P}=0.04$ by binomial test) and at the back compared with the sides $(\mathrm{P}=0.01)$ in actively moving cells (Fig. $3 \mathrm{~d}$ ). Cell F showed most activity at the back, presumably reflecting strong uropod contraction.

In cells undergoing TEM, we also observed RhoA activation in filopodia (Fig. 3 c) extending from the leading edge beneath EC (Fig. 3 e). Similar localization of active RhoA was observed in T cells fixed during TEM (Fig. S2 a). The lamellal puncta of high RhoA activity resemble the localization of LFA-1 (Fig. 2 a and Fig. 3 f; Smith et al., 2005; Stanley et al., 2008), and thus, could represent regions of T cell adhesion to ICAM-1 on the apical plasma membrane of EC and on the basal membrane after TEM (Millán et al., 2006). FRET efficiency was not dependent on the probe concentration (Fig. S2, e and f). As a control, we used a dominant-negative RhoA T19N Raichu probe (Yoshizaki et al., 2003), which had a much lower FRET efficiency compared with wild-type RhoA (Fig. S2, b and c; and Video 9). Expression of the Raichu probe did not detectably affect membrane dynamics, as determined by analysis of kymographs produced from videos of cells crawling on EC, nor did it affect cell polarity or TEM (Fig. S2, g-i).

RhoA activity is associated with both membrane extension and retraction events at the leading edge

The RhoA activity at the leading edge of T cells on EC was surprising because in neutrophil-like cells migrating in $2 \mathrm{D}$, RhoA was reported to be active mainly in the uropod (Wong et al., 2006), although occasional puncta of activity were observed near the front. However, RhoA is active in lamellipodia of fibroblasts, HeLa and MDCK cells, and in growth cones of neuroblastoma cells, where it is associated with plasma membrane protrusion (Kurokawa and Matsuda, 2005; Nakamura et al., 2005; Pertz et al., 2006; Picard et al., 2009). Therefore, we investigated how RhoA affected membrane dynamics. The leading edge and uropod of control $\mathrm{T}$ cells typically moved forward at the same constant rate with membrane ruffles at the front of the lamellipodium (Fig. 4, a and b). In most RhoA-depleted cells, the two or more long protrusions were stable except for some extension and retraction at the tips (Fig. 4 c). Kymographs were produced to examine membrane dynamics on these processes (Fig. 4, d and e). Slight lateral movement of the narrow protrusions meant that a continuous kymograph could not be performed over the same time course as control cells, and thus, protrusion dynamics were examined in four consecutive time frames. The tips of protrusions in RhoA-depleted cells extended much slower than lamellipodia of control cells, and only very rarely ruffled. However, we observed some membrane ruffling and lamellipodia along the protrusion perpendicular to the direction of extension (Fig. 4, f and g; and Video 4). In addition, local constrictions were observed along many protrusions, which is suggestive of myosin-mediated contraction. Protrusion and contraction are thus delocalized in the absence of RhoA and occur along protrusions rather than at the ends.
It has been hypothesized that RhoA could act at the leading edge to stimulate actin polymerization via mDia proteins and/or actomyosin contraction via ROCKs and phosphorylated myosin light chain (p-MLC) (Vicente-Manzanares and SanchezMadrid, 2004). Given the narrow protrusions and reduced protrusion rate after RhoA depletion, we examined whether changes in leading edge membrane dynamics were associated with RhoA activity (Fig. 4 h). We observed frequent transient localized activation of RhoA at the leading edge of T cells crawling on EC (Fig. S3, a-c), which was followed by either membrane extension $(52 \pm 7 \%$ SEM of discrete RhoA activity events) or retraction $(36 \pm 4 \% \mathrm{SEM})$. The remainder $(12 \pm 3 \% \mathrm{SEM})$ were not associated with extension or retraction in the next frame, although $40 \%$ of these were followed by membrane extension or retraction within $90 \mathrm{~s}$. These results imply that RhoA contributes to both extension and retraction events at the leading edge during migration, presumably acting via different effector complexes. Indeed, RhoA effectors involved in extension and retraction localize to the leading edge of migrating $\mathrm{T}$ cells, including mDia1 (Fig. 4 i; Vicente-Manzanares et al., 2003), low ROCK1 levels (Smith et al., 2003), myosin IIA (Rey et al., 2002), and p-MLC in over half of polarized cells (Fig. 4 j and Fig. S3 d), albeit often at lower intensity than at the rear.

\section{Roles of ROCKs and the RhoGEF GEF-H1 in the uropod}

Consistent with the RhoA activation we observe during contraction in the uropod, p-MLC staining was enriched in the uropod of both crawling and transmigrating T cells (Fig. $4 \mathrm{j}$ and Fig. S3 d), and RhoA-depleted cells had reduced p-MLC protein levels (Fig. 5 a), indicating that RhoA-mediated uropod contraction is involved in TEM. In T cells, ROCK inhibitors affect migration on ICAM-1 and fibronectin (Vicente-Manzanares et al., 2002; Smith et al., 2003); therefore, we investigated the role of ROCKs during crawling on EC and TEM by knocking down ROCK1 and ROCK2 with siRNAs (Fig. S3 e) or treating cells with the ROCK inhibitor Y27632. The main phenotype induced by ROCK knockdown or inhibition was long tails at the rear (Fig. 5, b and c). Many Y27632-treated cells had lateral protrusions along these tails (Fig. 5 d ), similar to those observed in RhoA knockdown cells (Fig. 2 a), indicating that ROCKs act both to mediate uropod contraction and suppress lateral protrusions (Worthylake et al., 2001; Vicente-Manzanares et al., 2002; Smith et al., 2003; Worthylake and Burridge, 2003). Interestingly, a significant increase in nonpolarized cells with two or more protrusions was observed in Y27632-treated cells, although this was not as high as with RhoA knockdown (Fig. 5 c). We then compared how protrusions formed in RhoA knockdown and Y27632-treated cells. Most RhoA-depleted cells formed protrusions by extension events from a rounded cell body (Fig. 5 e and Video 10). In contrast, most protrusions in Y27632-treated cells arose either through a tail retraction defect (40\% of cells with two protrusions) in which a cell with a long tail changed direction and formed a second tail at the opposite side or through a tail retraction defect and extension of a thin protrusion at the front (37.5\%; Video 10). The extension of thin protrusions could reflect a requirement for RhoA/ROCK-mediated 

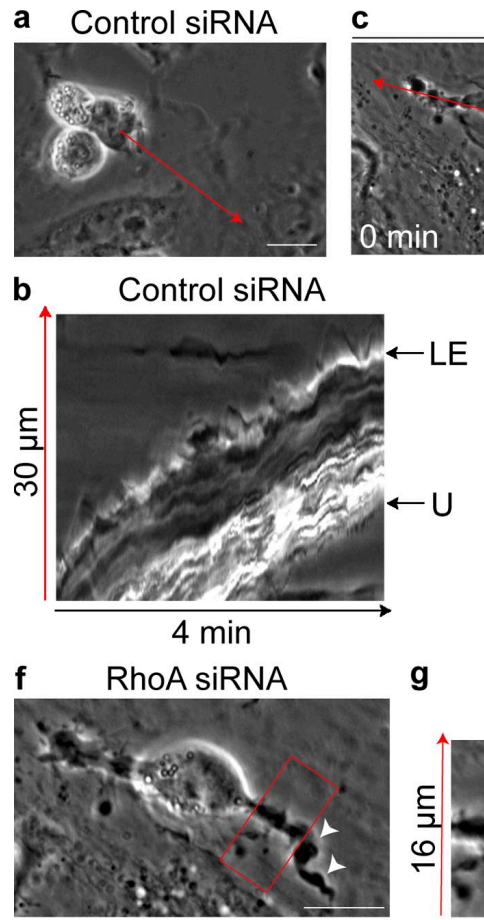

g
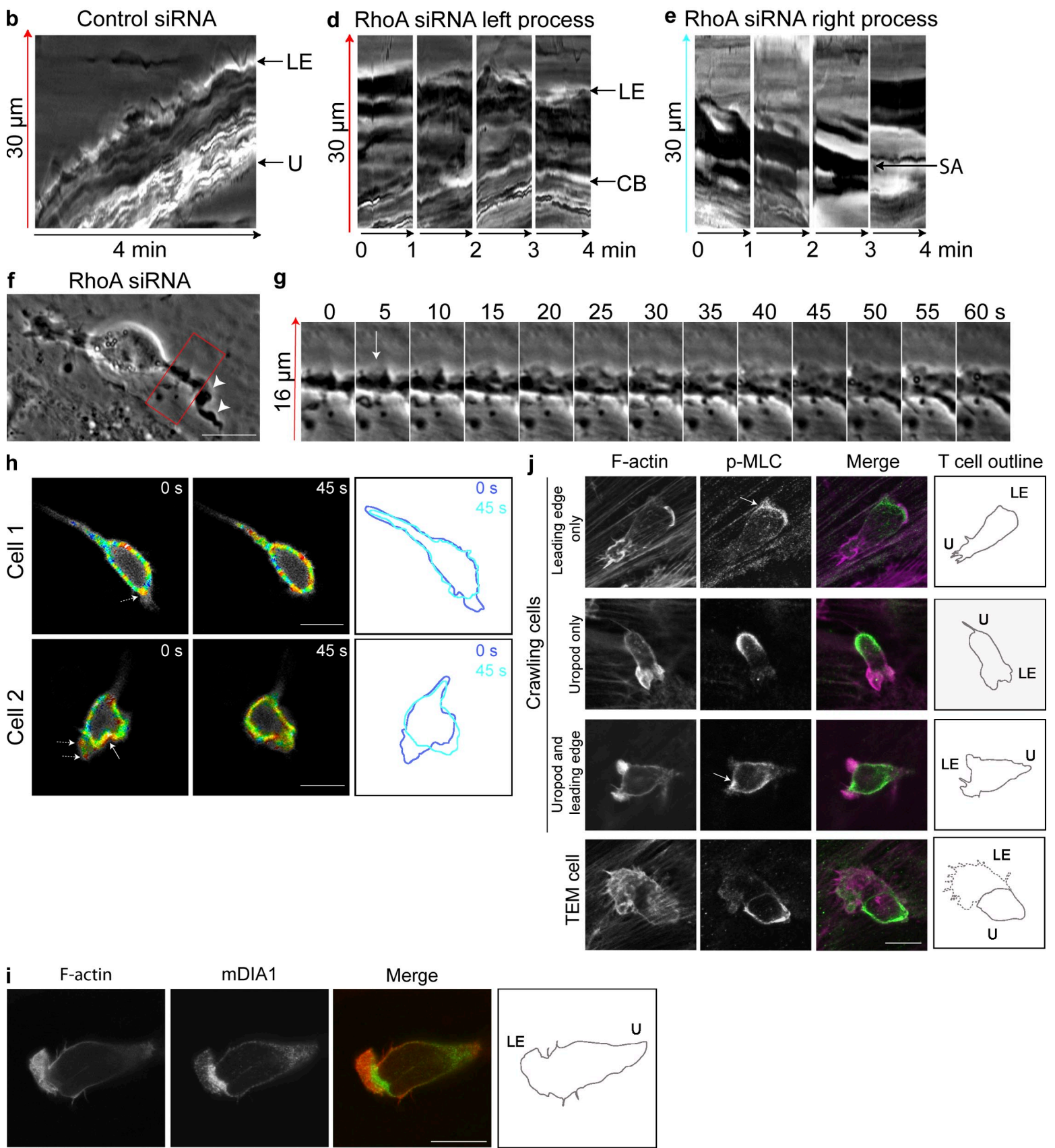

Figure 4. RhoA activity is associated with both extension and retraction of the membrane at the leading edge. (a-g) Images and kymographs (of regions marked with red/blue arrows) from time-lapse videos. (f and g) RhoA knockdown cell images show ruffling (starting at arrow; $5 \mathrm{~s}$ ) perpendicular to long extension. (f) Arrowheads, phase dark spherical accumulations (SA). Boxed region in $f$ is magnified in $g$. (h) Overlays of consecutive time-lapse images of RhoA Raichu probe lifetime (color scale) and probe localization on plasma membrane (white). Cell 1 shows RhoA activity before membrane retraction, and cell 2 shows before both retraction and extension. Solid arrow, extension; dashed arrows, retraction. Cell outlines show 0- (dark blue) and 45-s (light blue) frames. (i) mDIAl and F-actin localization in CEM cells on EC imaged by STED microscopy. (i) p-MLC and F-actin localization in CEM cells after 30 min on EC. Arrows, p-MLC at leading edge; dashed line, transmigrated region. LE, leading edge; $U$, uropod; $C B$, cell body; SA, phase dark spherical accumulations along protrusions. Bars, $10 \mu \mathrm{m}$. 
Figure 5. Roles of ROCKs and GEF-H 1 in T cell polarity on EC. (a) p-MLC levels in CEM cells. GAPDH, protein loading. Representative of four experiments. (b and c) Polarity quantification after $30 \mathrm{~min}$ on $\mathrm{EC}$ for siRNA-transfected $(48 \mathrm{~h})$ or Y27632-treated (10-min preincubation and continued presence) CEM cells (b, $n=3 ; c, n=5 ; 100$ cells/experiment). (d) Localization of F-actin, $\alpha$-tubulin, and ICAM-3 after $30 \mathrm{~min}$ on EC in Y27632-treated cells (10-min preincubation and continued presence). Arrows, long tails; arrowheads, lateral protrusions. (e) Process of CEM cell protrusion formation on EC determined from 1-h timelapse videos $(n=40$ cells $/$ condition; three experiments). (f) GEF-HI localization in CEM cells after 30 min on EC. LE, leading edge; $U$, uropod. (g) CEM cell length after $30 \mathrm{~min}$ on EC ( $n=3 ; 50$ cells/condition/experiment). ${ }^{*}, \mathrm{P}<0.05 ;{ }^{*}, \mathrm{P}<0.01$. Error bars indicate mean \pm SEM. Bars, $10 \mu \mathrm{m}$.

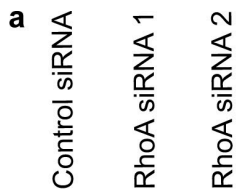

MLC2 (20kD)

$\mathrm{p}-\mathrm{MLC}(20 \mathrm{kD})$

RhoA (21kD)

GAPDH (36kD)
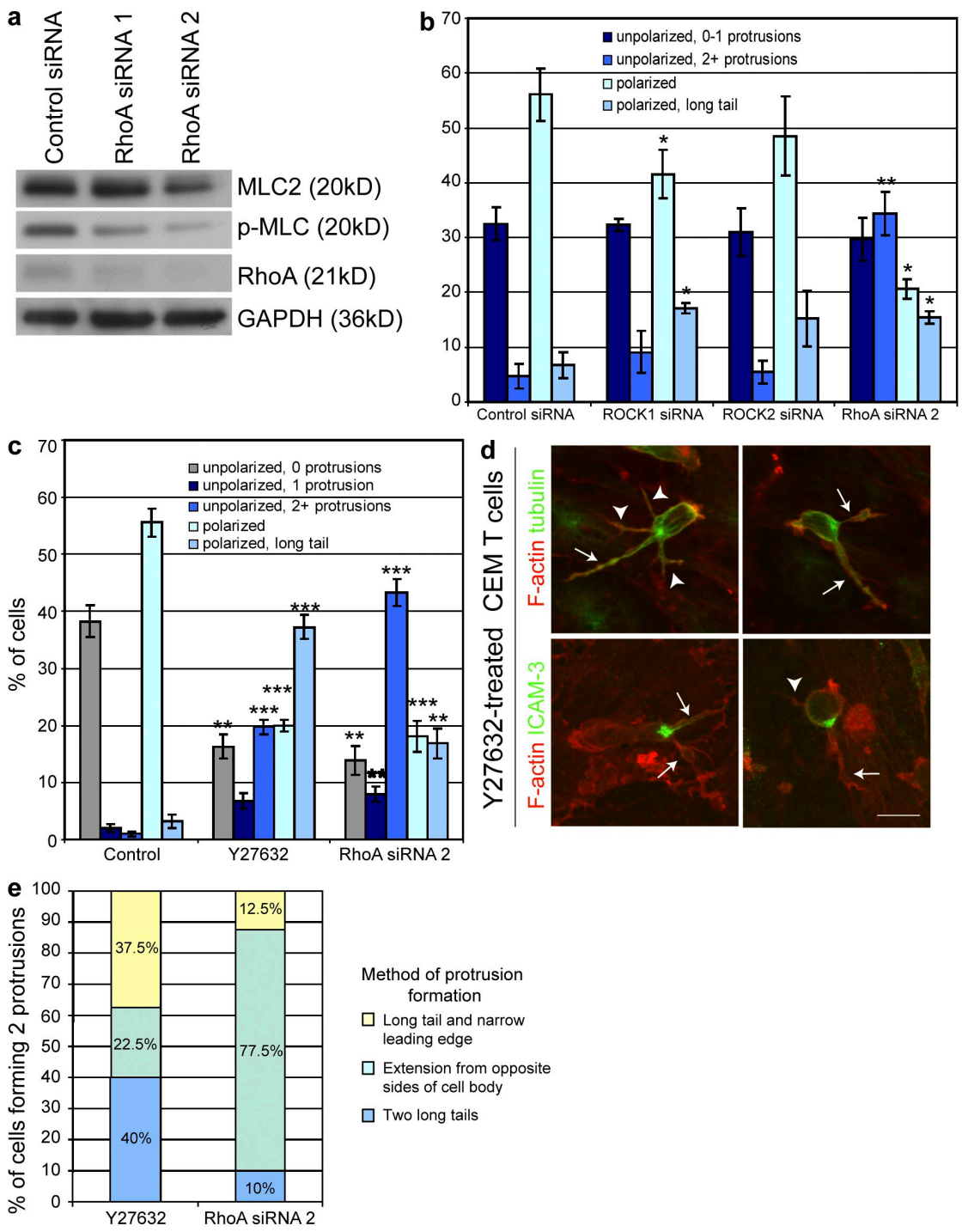

Method of protrusion formation

$\square$ Long tail and narrow leading edge

$\square$ Extension from opposite sides of cell body

$\square$ Two long tails
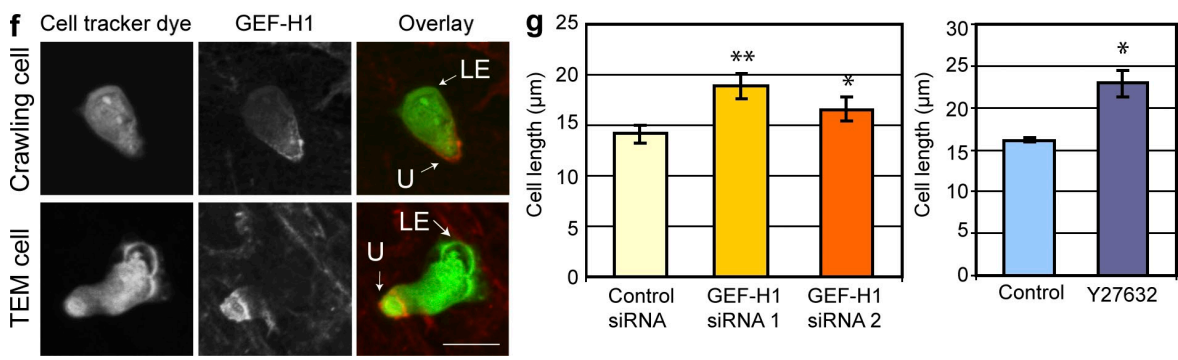

retraction events to form a broad lamellipodium. However, siRNA-mediated knockdown of ROCK1 and ROCK2 did not inhibit TEM as much as RhoA knockdown (Fig. S3 f), and thus, in addition to RhoA/ROCK-mediated contraction in the uropod and lamellipodium, it is likely that RhoA protrusive activity in the lamellipodia is important for TEM.

The localization of the RhoGEF GEF-H1 was investigated because it was previously reported to mediate RhoA activation at the leading edge of HeLa cells (Nalbant et al., 2009). In contrast, GEF-H1 localization was restricted to the uropod of crawling and transmigrating T cells (Fig. $5 \mathrm{f}$ and Fig. S3 g), suggesting that its function is to activate RhoA locally at the rear. Supporting this hypothesis, siRNA-mediated knockdown of GEF-H1 increased the length of T cells on EC, similar to ROCK inhibitor treatment, but did not induce thin protrusions or loss of the lamellipodium (Fig. $5 \mathrm{~g}$ and Fig. S3 h). GEF-H1 can activate RhoA-mediated myosin II-based contraction after its release from depolymerizing microtubules (Chang et al., 2008). We have recently found that ROCKs destabilize microtubules in T cells (Takesono et al., 2010), and thus, RhoA/ROCK signaling in the uropod could establish a positive feedback loop by promoting GEF-H1 release from microtubules, which in turn would increase RhoA activity.

Our data indicate that RhoA has at least three functions during T cell TEM: at the leading edge, it is required for both membrane protrusion and retraction, each of which contributes to the formation and extension of lamellipodia on and under 
$\mathrm{EC}$, and in the uropod, it is required for actomyosin-based contraction via ROCKs. We predict that RhoA activation for these three functions is independently regulated by different GEFs in response to the unique microenvironments in each cellular region. Furthermore, our data indicate that RhoA is locally activated, perhaps by a further GEF (Francis et al., 2006), at adhesion-like structures in the lamella.

\section{Materials and methods}

\section{Reagents}

The following antibodies and reagents were used from the indicated sources: mouse anti-Racl (clone 23A8) and rabbit anti-Rac2 (Millipore), rabbit anti-Rac3 (provided by A. Cox, University of North Carolina at Chapel Hill, Chapel Hill, NC), rabbit anti-RhoA (26C4), anti-RhoB, goat anti-RhoC (Santa Cruz Biotechnology, Inc.), sheep anti-RhoF (provided by H. Mellor, University of Bristol, UK), mouse anti-RhoJ, rabbit anti-RhoQ, goat anti-RhoU, rabbit anti-RhoBTB 1, anti-ICAM-3 antibody (Abcam), RhoH antibody (provided by D. Williams, Children's Hospital, Boston, MA), rabbit anti-Cdc42 and anti-pS19-MLC (Cell Signaling Technology), mouse antiRhoE as previously described (Riento et al., 2003), mouse anti-RhoG (provided by J. Meller and M. Schwartz, University of Virginia, Charlottesville, VA; Meller et al., 2008), mouse anti-ROCK1 and ROCK2, mouse antimDIA1 (BD), FITC-conjugated anti- $\alpha$-tubulin antibody (clone DMIA; Sigma-Aldrich), mouse anti-GEF-HI (provided by M. Balda, University College London, UK), Alexa Fluor 546- and 488-conjugated phalloidin (Invitrogen), recombinant human SDF-1 $\alpha$ (CXCL12), ICAM-1/Fc chimera, TNF (R\&D Systems), and fibronectin (Sigma-Aldrich).

\section{Cell culture}

CCRF-CEM T cells (CEM), T lymphoblasts, and human umbilical vein ECs (HUVECs) were cultured or purified and used as previously described (Millán et al., 2006; Takesono et al., 2010). HUVECs were stimulated with $10 \mathrm{ng} / \mathrm{ml}$ TNF for $16 \mathrm{~h}$ before experiments.

\section{siRNA and plasmid transfection}

Two different siRNAs or pools of four siRNAs (Thermo Fisher Scientific) were used as indicated. Proliferating CEM cells $\left(0.5 \times 10^{6}\right)$ were transiently transfected with $1.2 \mu \mathrm{M}$ siRNA or up to $20 \mu \mathrm{g}$ plasmid DNA using a nucleofector (Lonza) according to the manufacturer's instructions, and used for experiments after siRNA transfection after $48-72 \mathrm{~h}$ or after plasmid DNA transfection after $24 \mathrm{~h}$.

\section{Quantitative PCR}

Extracted mRNA was converted to cDNA and quantified using Primer Design SYBR green qPCR mastermix and primers (ABI PRISM 7000; Applied Biosystems). Primer sets were validated for amplification efficiency of 90-1 10\%. Relative mRNA expression was determined by comparison with the reference gene, GAPDH, using the Pfaffl method (Pfaffl, 2001).

\section{TEM in Transwell assays}

HUVECs were seeded onto fibronectin-coated 5-um Transwell filters or glass coverslips and grown to confluence, stimulated with TNF, and incubated for $15 \mathrm{~min}$ with $300 \mathrm{ng} / \mathrm{ml} \mathrm{SDF}-1 \alpha$ and washed. For time-lapse and immunofluorescence assays, the medium was replaced with CEM cells in medium. For Transwell assays, the medium was replaced with $200 \mu$ CEM cell medium containing $1.5 \times 10^{5} \mathrm{CEM}$ cells, and $600 \mu \mathrm{l}$ CEM cell medium containing $30 \mathrm{ng} / \mathrm{ml} \mathrm{SDF-1} \alpha$ was added to the lower chamber. After $2 \mathrm{~h}$, transmigrated cells were counted using a Casy counter.

\section{Time-lapse microscopy, cell tracking, and analysis}

CEM cells were added to confluent TNF-stimulated HUVECs, and images were acquired at $37^{\circ} \mathrm{C}$ and $5 \% \mathrm{CO}_{2}$ using a time-lapse microscope (Eclipse TE 2000-E; Nikon) with a charge-coupled device camera (ORCA; Hamamatsu Photonics). Images were acquired every minute for $1-2 \mathrm{~h}$ using a $20 \times 0.45$ NA objective or for high magnification $(90 \times)$ every second using a $60 \times 1.4$ NA oil immersion objective with 1.5x magnification. Images were acquired using MetaMorph software (MDS Analytical Technologies), and cells were tracked using ImageJ software (National Institutes of Health) and analyzed using the Chemotaxis plug in. Kymographs were produced in MetaMorph from 90x magnification videos. The method of protrusion formation was quantified from time-lapse videos. CEM cells were allowed to settle on HUVECs after which Y27632 was added, or RhoA knockdown cells were added to HUVECs and allowed to settle. Time-lapse videos were acquired for a minimum of $1 \mathrm{~h}$, capturing 1 frame/min. Protrusions were classified based on having (a) a defect in tail retraction in combination with a narrow leading edge (long tail and narrow leading edge), (b) a defect in tail retraction followed by a reverse in migratory direction and formation of a second long tail (two long tails), or (c) an extension of protrusions from a rounded cell body (extension from opposite sides of cell body).

\section{FLIM/FRET assays}

The use of the Raichu RhoA probe and negative control RhoA-T19N probe has previously been described (Yoshizaki et al., 2003). Probes were modified to express GFP and mRFP as described for Racl and Cdc42 biosensors (Makrogianneli et al., 2009). Multiphoton time-correlated single photon counting FLIM was performed to quantify RhoA biosensor FRET. An inverted microscope (TE2000E; Nikon) combined with an in-house scanner (Peter et al., 2005) and either a solid-state-pumped (Verdi; Coherent) or self mode-locked Ti:Sapphire laser (Mira or Chameleon; Coherent) for multiphoton excitation was used. All images were acquired at a suitable spatial and time resolution to provide enough photon arrival times for accurate fluorescence decay fitting, while avoiding detector pile up, and analyzed by performing a single-exponential pixel fit in time-resolved image analysis software (TRI2; Peter et al., 2005; Barber et al., 2009; Makrogianneli et al., 2009; Carlin et al., 2010).

For fixed samples, transfected cells were added to HUVECs for $30 \mathrm{~min}$, fixed with $4 \%$ paraformaldehyde, mounted using fluorescent mounting media (Dako), and imaged at room temperature using a $40 \times 1.4 \mathrm{NA}$ oil immersion objective onto a charge-coupled device camera (Orca; Hamamatsu Photonics). For live samples, transfected cells were allowed to settle on HUVECs grown in microincubator plates (Smart Slide; Labtech International) to maintain them at $37^{\circ} \mathrm{C}$ and $5 \% \mathrm{CO}_{2}$. Time-resolved images were acquired over $30 \mathrm{~s}$ every $45 \mathrm{~s}$, to provide enough photons for fitting without saturating the detector; time resolution was reduced to 64 bins to optimize this. FRET efficiency is defined as 1 - (fluorescence lifetime of donor and acceptor [GFP + RFP]/fluorescence lifetime of donor alone [GFP]). GFP alone lifetimes were measured at 2.25 (fixed) and $2.35 \mathrm{~ns}$ (live). Images were analyzed using TRI2 (Peter et al., 2005; Barber et al., 2009; Makrogianneli et al., 2009; Carlin et al., 2010) and ImageJ.

Localization analysis of RhoA activity was performed by thresholding the intensity image and finding the cell centroid. To determine the movement magnitude, the position of the maxima along radial lines was calculated for each angle, and the mean was compared with the mean of the previous time frame. The front of the leading edge was calculated by extrapolating the movement vector from the center of the cell. The outermost three pixels of the FLIM-FRET data were selected, thresholded, and categorized as front, back, or sides with each segment being $90^{\circ}$ and the center of the leading edge being the center of the front segment. $0 \pm 45^{\circ}$ represented the front of the cell, the two sides were $45-135^{\circ}$ and -45 to $-135^{\circ}$, and the back was $180 \pm$ $45^{\circ}$. If the movement magnitude was $>2$ pixels, it was considered possible to make a meaningful front/back assignment. The mean FLIM/FRET value in each segment was calculated and compared. These tests were performed for those cells in which the changes in cell shape from frame to frame were sufficiently small such that movement could be consistently categorized. Data were analyzed using a binomial statistical test.

To represent RhoA activity localization graphically, the front of the leading edge was identified as described in the previous paragraph. In the frames in which the movement magnitude was $<2$ pixels, the position of the front of the leading edge from the previous frame was used. The FLIM image was split into angular segments with the RhoA activation of the outer 3 pixels being averaged. The movement of each angular segment was measured relative to the previous frame (note that this will include both the effects of global movement and shape changes). The mean value of the RhoA activation in each segment was calculated after thresholding.

\section{Immunofluorescence}

CEM T cells or T lymphoblasts were added to HUVECs and fixed after $30 \mathrm{~min}$ for $15 \mathrm{~min}$ with $4 \%$ paraformaldehyde or $10 \%$ trichloroacetic acid (RhoA localization). Cells were permeabilized with $0.1 \%$ Triton X-100, blocked with $1 \%$ BSA, and incubated with FITC-conjugated anti- $\alpha$-tubulin (1:200), anti-Cd 1 la (1:50), anti-ICAM-3 (1:50), anti-RhoA (1:50), anti-p-MLC (1:50), anti-mDIA1 (1:100), and/or anti-p-ERM (1:100) antibodies for $1 \mathrm{~h}$ followed by Alexa Fluor 488- or 546-conjugated anti-rabbit or anti-mouse IgG secondary antibodies (1:200) and/or Alexa Fluor 546- or 647conjugated phalloidin $11: 200)$ for $1 \mathrm{~h}$, and mounted using fluorescent mounting medium (Dako). Images were acquired at room temperature using a confocal microscope (LSM 510 META; Carl Zeiss, Inc.) with three single 
photomultiplier tube detectors mounted on a inverted microscope (AxioObserver Z1; Carl Zeiss, Inc.) using a 40× 1.3 NA oil immersion objective (Carl Zeiss, Inc.). Images were acquired using Zen software (Carl Zeiss, Inc.) and processed using Zen and Photoshop (Adobe). For stimulated emission depletion (STED) microscopy, CEM T cells were fixed 30 min after addition to EC, permeabilized, blocked, and stained with Atto $647 \mathrm{~N}$-phalloidin (Sigma-Aldrich) to localize F-actin and anti-mDIAl antibodies and coverslips mounted using mounting media (ProLong; Invitrogen). Cells were imaged at room temperature with a 100 1.4 NA oil immersion objective (Leica) using an STED microscope (TCS SP5 II; Leica) in STED mode (F-actin) or confocal mode (mDIA1).

For quantification of polarization, polarized CEM cells (labeled with Cell Tracker dye) were defined as cells with a morphologically polarized shape, F-actin accumulation at the front, and the MTOC (identified by $\alpha$-tubulin staining) behind the nucleus. 100 cells/coverslip were counted in a minimum of three independent experiments.

For quantification of CEM cell length, siRNA-transfected CEM cells were labeled with Cell Tracker dye $72 \mathrm{~h}$ after transfection and added to EC. After $30 \mathrm{~min}$, cells were fixed and stained for $\alpha$-tubulin and GEF-HI . For Y27632 experiments, Cell Tracker dye-labeled cells were preincubated with Y27632 for $10 \mathrm{~min}$ and added to EC in the continued presence of $Y 27632$, fixed after $30 \mathrm{~min}$, and stained for $\alpha$-tubulin and F-actin. Confocal z-stack images were acquired and used to measure the cell length of crawling CEM T cells and to measure GEF-HI staining intensity to identify GEF-H1-depleted cells.

\section{Statistical analysis}

Statistical significance was determined using a two-way paired Student's $t$ test unless otherwise indicated.

\section{Online supplemental material}

Fig. S1 shows expression and roles of Rho GTPases in T cell TEM. Fig. S2 shows localization of RhoA activity in T cells migrating on and across ECs. Fig. S3 shows the roles of RhoA, ROCKs, and GEF-H1 in T cells. Video 1 shows that RhoA knockdown inhibits T cell TEM. Video 2 shows protrusion dynamics in a control T cell. Video 3 shows tail extension in a RhoAdepleted T cell. Video 4 shows protrusion dynamics in a RhoA-depleted T cell. Videos 5 and 6 show localization of RhoA activity in crawling T cells. Videos 7 and 8 show localization of RhoA activity in transmigrating T cells. Video 9 shows localization of the control dominant-negative RhoA probe in a crawling T cell. Video 10 shows effects of $Y 27632$ on T cell protrusions. Online supplemental material is available at http://www.jcb .org/cgi/content/full/jcb.201002067/DC1.

We thank A. Cox, M. Balda, H. Mellor, D. Williams, J. Meller, M. Schwartz, K. Makrogianneli, and A. Thrasher for reagents and A. Takesono, P. Bhavsar, and M. Arno for discussions and advice.

This work was funded by the Medical Research Council (A.J. Ridley), the Bettencourt-Schueller Foundation (A.J. Ridley), the Leukaemia Research Fund (A.J. Ridley), Engineering and Physical Sciences Research Council (L. Carlin; T. Ng), and the Richard Dimbleby Cancer Fund (T. Ng).

Submitted: 12 February 2010

Accepted: 22 July 2010

\section{References}

Alblas, J., L. Ulfman, P. Hordijk, and L. Koenderman. 2001. Activation of Rhoa and ROCK are essential for detachment of migrating leukocytes. Mol. Biol. Cell. 12:2137-2145.

Barber, P.R., S.M. Ameer-Beg, J. Gilbey, L.M. Carlin, M. Keppler, T.C. Ng, and B. Vojnovic. 2009. Multiphoton time-domain fluorescence lifetime imaging microscopy: practical application to protein-protein interactions using global analysis. J. R. Soc. Interface. 6:S93-S105. doi:10.1098/ rsif.2008.0451.focus

Barreiro, O., M. Yanez-Mo, J.M. Serrador, M.C. Montoya, M. VicenteManzanares, R. Tejedor, H. Furthmayr, and F. Sanchez-Madrid. 2002. Dynamic interaction of VCAM-1 and ICAM-1 with moesin and ezrin in a novel endothelial docking structure for adherent leukocytes. J. Cell Biol. 157:1233-1245. doi:10.1083/jcb.200112126

Bolis, A., S. Corbetta, A. Cioce, and I. de Curtis. 2003. Differential distribution of Rac1 and Rac3 GTPases in the developing mouse brain: implications for a role of Rac3 in Purkinje cell differentiation. Eur. J. Neurosci. 18:2417-2424. doi:10.1046/j.1460-9568.2003.02938.x

Boulter, E., R. Garcia-Mata, C. Guilluy, A. Dubash, G. Rossi, P.J. Brennwald, and K. Burridge. 2010. Regulation of Rho GTPase crosstalk, degradation and activity by RhoGDI1. Nat. Cell Biol. 12:477-483. doi:10.1038/ ncb2049

Boureux, A., E. Vignal, S. Faure, and P. Fort. 2007. Evolution of the Rho family of ras-like GTPases in eukaryotes. Mol. Biol. Evol. 24:203-216. doi:10.1093/ molbev/msl145

Carlin, L.M., K. Makrogianneli, G. Fruhwirth, and T. Ng. 2010. Visualizing signalling in immune cells. In T-Cell Trafficking: Methods and Protocols F. M. Marelli-Berg and S. Nourshargh, editors. Springer-Verlag New York Inc., New York. 97-113.

Carman, C.V., and T.A. Springer. 2004. A transmigratory cup in leukocyte diapedesis both through individual vascular endothelial cells and between them. J. Cell Biol. 167:377-388. doi:10.1083/jcb.200404129

Carman, C.V., C.D. Jun, A. Salas, and T.A. Springer. 2003. Endothelial cells proactively form microvilli-like membrane projections upon intercellular adhesion molecule 1 engagement of leukocyte LFA-1. J. Immunol. 171:6135-6144.

Chang, Y.C., P. Nalbant, J. Birkenfeld, Z.F. Chang, and G.M. Bokoch. 2008. GEF-H1 couples nocodazole-induced microtubule disassembly to cell contractility via RhoA. Mol. Biol. Cell. 19:2147-2153. doi:10.1091/mbc .E07-12-1269

Filippi, M.D., K. Szczur, C.E. Harris, and P.Y. Berclaz. 2007. Rho GTPase Rac1 is critical for neutrophil migration into the lung. Blood. 109:1257-1264. doi: 10.1182/blood-2006-04-017731

Francis, S.A., X. Shen, J.B. Young, P. Kaul, and D.J. Lerner. 2006. Rho GEF Lsc is required for normal polarization, migration, and adhesion of formyl-peptide-stimulated neutrophils. Blood. 107:1627-1635. doi:10 .1182/blood-2005-03-1164

Gérard, A., R.A. van der Kammen, H. Janssen, S.I. Ellenbroek, and J.G. Collard. 2009. The Rac activator Tiam1 controls efficient T-cell trafficking and route of transendothelial migration. Blood. 113:6138-6147. doi:10.1182/ blood-2008-07-167668

Giagulli, C., E. Scarpini, L. Ottoboni, S. Narumiya, E.C. Butcher, G. Constantin, and C. Laudanna. 2004. RhoA and zeta PKC control distinct modalities of LFA- 1 activation by chemokines: critical role of LFA- 1 affinity triggering in lymphocyte in vivo homing. Immunity. 20:25-35. doi:10.1016/ S1074-7613(03)00350-9

Hajdo-Milasinović, A., S.I. Ellenbroek, S. van Es, B. van der Vaart, and J.G. Collard. 2007. Rac1 and Rac3 have opposing functions in cell adhesion and differentiation of neuronal cells. J. Cell Sci. 120:555-566. doi:10.1242/ jcs.03364

Heasman, S.J., and A.J. Ridley. 2008. Mammalian Rho GTPases: new insights into their functions from in vivo studies. Nat. Rev. Mol. Cell Biol. 9:690-701. doi: $10.1038 / \mathrm{nrm} 2476$

Jaffe, A.B., and A. Hall. 2005. Rho GTPases: biochemistry and biology. Annu. Rev. Cell Dev. Biol. 21:247-269. doi:10.1146/annurev.cellbio.21.020604 .150721

Kurokawa, K., and M. Matsuda. 2005. Localized RhoA activation as a requirement for the induction of membrane ruffling. Mol. Biol. Cell. 16:42944303. doi:10.1091/mbc.E04-12-1076

Makrogianneli, K., L.M. Carlin, M.D. Keppler, D.R. Matthews, E. Ofo, A. Coolen, S.M. Ameer-Beg, P.R. Barber, B. Vojnovic, and T. Ng. 2009. Integrating receptor signal inputs that influence small Rho GTPase activation dynamics at the immunological synapse. Mol. Cell. Biol. 29: 2997-3006. doi:10.1128/MCB.01008-08

Matsui, T., S. Yonemura, S. Tsukita, and S. Tsukita. 1999. Activation of ERM proteins in vivo by Rho involves phosphatidyl-inositol 4-phosphate 5-kinase and not ROCK kinases. Curr. Biol. 9:1259-1262. doi:10.1016/ S0960-9822(99)80508-9

Meller, J., L. Vidali, and M.A. Schwartz. 2008. Endogenous RhoG is dispensable for integrin-mediated cell spreading but contributes to Rac-independent migration. J. Cell Sci. 121:1981-1989. doi:10.1242/jcs.025130

Millán, J., L. Hewlett, M. Glyn, D. Toomre, P. Clark, and A.J. Ridley. 2006. Lymphocyte transcellular migration occurs through recruitment of endothelial ICAM-1 to caveola- and F-actin-rich domains. Nat. Cell Biol. 8:113-123. doi:10.1038/ncb1356

Nakamura, T., K. Aoki, and M. Matsuda. 2005. FRET imaging in nerve growth cones reveals a high level of RhoA activity within the peripheral domain. Brain Res. Mol. Brain Res. 139:277-287. doi:10.1016/j.molbrainres.2005.05.030

Nalbant, P., Y.C. Chang, J. Birkenfeld, Z.F. Chang, and G.M. Bokoch. 2009. Guanine nucleotide exchange factor-H1 regulates cell migration via localized activation of RhoA at the leading edge. Mol. Biol. Cell. 20:40704082. doi:10.1091/mbc.E09-01-0041

Pertz, O., L. Hodgson, R.L. Klemke, and K.M. Hahn. 2006. Spatiotemporal dynamics of RhoA activity in migrating cells. Nature. 440:1069-1072. doi:10 .1038 /nature04665

Peter, M., S.M. Ameer-Beg, M.K. Hughes, M.D. Keppler, S. Prag, M. Marsh, B. Vojnovic, and T. Ng. 2005. Multiphoton-FLIM quantification of the 
EGFP-mRFP1 FRET pair for localization of membrane receptor-kinase interactions. Biophys. J. 88:1224-1237. doi:10.1529/biophysj.104.050153

Pfaffl, M.W. 2001. A new mathematical model for relative quantification in realtime RT-PCR. Nucleic Acids Res. 29:e45. doi:10.1093/nar/29.9.e45

Phillipson, M., B. Heit, P. Colarusso, L. Liu, C.M. Ballantyne, and P. Kubes. 2006. Intraluminal crawling of neutrophils to emigration sites: a molecularly distinct process from adhesion in the recruitment cascade. J. Exp. Med. 203:2569-2575. doi:10.1084/jem.20060925

Picard, M., R.J. Petrie, J. Antoine-Bertrand, E. Saint-Cyr-Proulx, J.F. Villemure, and N. Lamarche-Vane. 2009. Spatial and temporal activation of the small GTPases RhoA and Rac1 by the netrin-1 receptor UNC5a during neurite outgrowth. Cell. Signal. 21:1961-1973.

Rey, M., M. Vicente-Manzanares, F. Viedma, M. Yáñez-Mó, A. Urzainqui, O. Barreiro, J. Vázquez, and F. Sánchez-Madrid. 2002. Cutting edge: association of the motor protein nonmuscle myosin heavy chain-IIA with the $\mathrm{C}$ terminus of the chemokine receptor CXCR4 in $\mathrm{T}$ lymphocytes. J. Immunol. 169:5410-5414.

Riento, K., R.M. Guasch, R. Garg, B. Jin, and A.J. Ridley. 2003. RhoE binds to ROCK I and inhibits downstream signaling. Mol. Cell. Biol. 23: 4219-4229. doi:10.1128/MCB.23.12.4219-4229.2003

Roberts, A.W., C. Kim, L. Zhen, J.B. Lowe, R. Kapur, B. Petryniak, A. Spaetti, J.D. Pollock, J.B. Borneo, G.B. Bradford, et al. 1999. Deficiency of the hematopoietic cell-specific Rho family GTPase Rac 2 is characterized by abnormalities in neutrophil function and host defense. Immunity. 10: 183-196. doi:10.1016/S1074-7613(00)80019-9

Sánchez-Madrid, F., and J.M. Serrador. 2009. Bringing up the rear: defining the roles of the uropod. Nat. Rev. Mol. Cell Biol. 10:353-359. doi:10 $.1038 / \mathrm{nrm} 2680$

Schenkel, A.R., Z. Mamdouh, and W.A. Muller. 2004. Locomotion of monocytes on endothelium is a critical step during extravasation. Nat. Immunol. 5:393-400. doi:10.1038/ni1051

Smith, A., M. Bracke, B. Leitinger, J.C. Porter, and N. Hogg. 2003. LFA-1induced T cell migration on ICAM-1 involves regulation of MLCK-mediated attachment and ROCK-dependent detachment. J. Cell Sci. 116:3123-3133. doi:10.1242/jcs.00606

Smith, A., Y.R. Carrasco, P. Stanley, N. Kieffer, F.D. Batista, and N. Hogg. 2005. A talin-dependent LFA-1 focal zone is formed by rapidly migrating T lymphocytes. J. Cell Biol. 170:141-151. doi:10.1083/jcb.200412032

Stanley, P., A. Smith, A. McDowall, A. Nicol, D. Zicha, and N. Hogg. 2008. Intermediate-affinity LFA-1 binds alpha-actinin-1 to control migration at the leading edge of the T cell. EMBO J. 27:62-75. doi:10.1038/sj .emboj.7601959

Szczur, K., H. Xu, S. Atkinson, Y. Zheng, and M.D. Filippi. 2006. Rho GTPase CDC42 regulates directionality and random movement via distinct MAPK pathways in neutrophils. Blood. 108:4205-4213. doi:10.1182/ blood-2006-03-013789

Takesono, A., S.J. Heasman, B. Wojciak-Stothard, R. Garg, and A.J. Ridley. 2010. Microtubules regulate migratory polarity through Rho/ROCK signaling in T cells. PLoS One. 5:e8774. doi:10.1371/journal.pone.0008774

Tybulewicz, V.L., and R.B. Henderson. 2009. Rho family GTPases and their regulators in lymphocytes. Nat. Rev. Immunol. 9:630-644. doi:10.1038/nri2606

van Buul, J.D., M.J. Allingham, T. Samson, J. Meller, E. Boulter, R. GarcíaMata, and K. Burridge. 2007. RhoG regulates endothelial apical cup assembly downstream from ICAM1 engagement and is involved in leukocyte trans-endothelial migration. J. Cell Biol. 178:1279-1293. doi:10 $.1083 /$ jcb. 200612053

Vestweber, D. 2007. Adhesion and signaling molecules controlling the transmigration of leukocytes through endothelium. Immunol. Rev. 218:178196. doi:10.1111/j.1600-065X.2007.00533.x

Vicente-Manzanares, M., and F. Sánchez-Madrid. 2004. Role of the cytoskeleton during leukocyte responses. Nat. Rev. Immunol. 4:110-122. doi:10.1038/ nri1268

Vicente-Manzanares, M., J.R. Cabrero, M. Rey, M. Pérez-Martínez, A. Ursa, K. Itoh, and F. Sánchez-Madrid. 2002. A role for the Rho-p160 Rho coiledcoil kinase axis in the chemokine stromal cell-derived factor-1alpha-induced lymphocyte actomyosin and microtubular organization and chemotaxis. J. Immunol. 168:400-410.

Vicente-Manzanares, M., M. Rey, M. Pérez-Martínez, M. Yáñez-Mó, D. Sancho, J.R. Cabrero, O. Barreiro, H. de la Fuente, K. Itoh, and F. SánchezMadrid. 2003. The RhoA effector mDia is induced during $\mathrm{T}$ cell activation and regulates actin polymerization and cell migration in T lymphocytes. J. Immunol. 171:1023-1034.

Wittchen, E.S. 2009. Endothelial signaling in paracellular and transcellular leukocyte transmigration. Front. Biosci. 14:2522-2545. doi:10.2741/3395

Wong, K., O. Pertz, K. Hahn, and H. Bourne. 2006. Neutrophil polarization: spatiotemporal dynamics of RhoA activity support a self-organizing mechanism. Proc. Natl. Acad. Sci. USA. 103:3639-3644. doi:10.1073/pnas .0600092103
Worthylake, R.A., and K. Burridge. 2003. RhoA and ROCK promote migration by limiting membrane protrusions. J. Biol. Chem. 278:13578-13584. doi: 10.1074/jbc.M211584200

Worthylake, R.A., S. Lemoine, J.M. Watson, and K. Burridge. 2001. RhoA is required for monocyte tail retraction during transendothelial migration. J. Cell Biol. 154:147-160. doi:10.1083/jcb.200103048

Yamauchi, A., C. Kim, S. Li, C.C. Marchal, J. Towe, S.J. Atkinson, and M.C. Dinauer. 2004. Rac2-deficient murine macrophages have selective defects in superoxide production and phagocytosis of opsonized particles. J. Immunol. 173:5971-5979.

Yoshizaki, H., Y. Ohba, K. Kurokawa, R.E. Itoh, T. Nakamura, N. Mochizuki, K. Nagashima, and M. Matsuda. 2003. Activity of Rho-family GTPases during cell division as visualized with FRET-based probes. J. Cell Biol. 162:223-232. doi:10.1083/jcb.200212049 